\title{
DESARROLLO LONGITUDINAL Y PONDERAL DEL LUCIO (ESOX lucius) EN UN AREA DE RECIENTE COLONIZACION: CUENCA DEL ESLA, NW ESPAÑA.
}

\author{
J. Domínguez y J.C. Pena \\ Dpto. dc Biología Animal. Universidad de Leóri. 24071 LEÓN. ESPAÑA. E.mail: dbajpa@unileon.es
}

Palabras clave: lucio. crecimiento, desarrollo pondcral, cuenca del Esla.

Keywords: pike, growth, length-weight equation, Esla Basin, Spain.

\begin{abstract}
LENCTH AND GROWTH DEVELOPMENT IN PIKE (Esox lucius) IN AN NEW COLONIZED AREA: ESLA BASIN (N.W. SPAIN).

The growth of pikc, Esox lucius, has been studied under natural conditions in the Esla basin (NW of Spain). The aiin of this work is to analyse the differences between this population and others.

A large data set was obtained during seven ycars period. The length-weight relationship and relative condition was calculated on 4.369 spccimens. Statistically significant differences bctween both sexes occurred in growth weigth, affected by the gonad cycle of inature fcrnales.

Scales of 1,658 pike from Esla basin were used for age and back-calculated growth deterininations. Pike growth was comparable with the slowest in others watcrs. We attempt to explain the rclationships between development in length and feedinp habits.
\end{abstract}

\section{INTRODUCCIÓN}

En 1949 se lleva a cabo la introducción dc huevos embrionados de lucio (GUTIERREZ-CALDERON, 1954) a partir dc los cuales se inicia la diseminación en numerosos ríos hasta los años 80 .

La primera fecha en que se registra esta especic en la cuenca del Esla es cn 1964 (PENA, 1986), convirtiéndose, a medida que asciende por los ríos de la cuenca, en un elemento distorsionador dc las coinunidades de peces establecidas, provocaiido un descenso importante en los efectivos de las poblaciones, bien por depredación o bien por desplazamiento (RINCON et al., 1990). La rápida ocupación de un gran número dc cursos fluviales evidencia el éxito conseguido.

El carácter truchero de muchas de las aguas de la cuenca confiere una dimensión diferente a la presencia del lucio respecto a cuencas fluviales de la mitad Sur de la Península Ibérica, donde la presencia de esta cspecie ha servido como alicicnte para la pesca recreativa.

Limnetica. 12 (2): 79-92 (1996)

(6) Asociación Española de Limología, Madrid. Spain
La oportiinidad de efectuar el control dc esta especie ha proporcionado el material de trabajo para poder tratar algunos aspectos de la biología del lucio cn una cuenca fluvial cerrada.

\section{MATERIAL Y METODOS}

\section{Zona de estudio}

Situada en la parte sur de la provincia de León y norte de la de Zamora el área de estudio incluye la zona colonizada por el lucio en la cuenca del Esla, aguas arriba del cmbalse de Ricobayo (Figura 1). La red hidrográfica de dicha cuenca está constituida por los ríos Esla, Porma, Moro, Órbigo, Tuerto, Jamuz, Eria, Cea, Tcra y los Arroyos de La Almucera, El Castrón, Retiierta, Regato y Bonul. De todos ellos sólo los arroyos y los ríos Cea, Eria y Moro, perinancecn sin rcgulai. El Esla está regulado en cabecera desde el año 1988, fecha posterior al periodo de muestreo, por el embalse de Riaño de $664 \mathrm{Hm}^{3}$ y en la parte inferior por el embalse de Ricobayo (1 $184 \mathrm{Hm}^{3}$ ). 


\section{Muestreo y tratamiento de datos}

El muestreo se ha realizado entre enero de 1982 y septiernbrc dc 1987, aunque la mayor parte de los ejemplares se capturaron enti-e febrero de 1986 y septiernbre de 1987. Se estudia un total de 4.369 ejemplares.La captura de los ejemplares se realizó mediante pesca eléctrica, trampas dc rcproductorcs y redes. La pesca eléctrica ha sido el método de elección complementado con trampas de reproductores en arroyos y accesos a lagunas y brazos muertos de río durante la época dc freza, entre los meses de febrero y mayo (Arroyo de la Almucera, A" del Castrón, Hospital de Orbigo, Marne, La Nora, Puente Villarentc-río Moro y Villafruela) y trasmallos de 3 crii de luz de malla desde barca.De cada ejemplar fue medida su Jongitud total, pesado -con precisión de $1 \mathrm{~mm}$ y 1 gr respectivamente- y se recogió una inucstra de escamas (BOET \& LE LOUARN. 1985) que posteriormente fueron limpiadas con hidróxido sódico al $4 \%$ y montadas, en seco, entre porta y cubre. La lectura de la edad y medición de los radios se efectuó con un lector de perfiles Nikon V-12 que permite una precisión de medida de $1 \mathrm{~m}$.Se efectuaron dos lecturas de cada muestra, eliininando aquellas en que se obtenían diferentes resultados, como aconsejan BOET \& LE LOUARN (1985). Cada ejemplar ha sido incluido en la clase correspondiente al número de annuli que presentaba.

Para la dcteriiiinación de la edad se efectuó la lectura y medición de escamas de un total de 1.658 lucios seleccionados de cntre el total de los utilizados en el estudio. Por dificultades dc lectura o de distribución de los annuli se han desechado el $4.76 \%$ dc las escamas seleccionadas.
Se estableció la relación entre el tamaño de la escama y la longitud del ejeinplar del que procedía utilizando el radio anterior (Ra) y el posterior (Rp), con la disposición del eje anteroposterior y se calculó el mejor ajuste. La longitud del ejeinplar más pequeño utilizado ha sido $73 \mathrm{~min}$, con el cuerpo totalmente cubierto de escamas.Para evaluar si la relación entrc el radio de la escaina y la longitud del ejemplar ofrecía diferencias en función del sexo de los individuos se realizó un análisis de covarianza (TAHACHNICK \& FIDELL, 1986) y para estimar las diferencias de longitud entre sexos para cada clase de edad se realizó un análisis de varianza (STEEL \& TORRIE, 1985).

El crecimiento se ha estimado inediante la ecuación de Voii Bertalanffy (BAGENAL \& TESCH, 1978) utilizando el prograina para ordenador persorial L.F.S.A.de SPARRE (1987).

Para evaluar si existían diferencias para las curvas de $V_{0}$ Bertalanffy calculadas para cada sexo se realizó el análisis de la suma de cuadrados de los rcsiduales según modelo propuesto por Ratkowsky para inodelos no lineales (CHEN et al., 1992).

La estimación de las constantes de la ecuación que relaciona la longitud y el peso se efectuó incdiantc regresióri por mínimos cuadrados (BAGENAL \& TESCH, 1978). Para detectar la variación debida al sexo y al mes dc captura de los ejernplares se empleó el análisis de covarianza; cuando existía más de una variable estas sc recodificaban para proceder a un análisis dc una sola vía (TABACHNICK \& FIDELL, 1986). Sc consideró un nivel de significación superior al 99\%.

\begin{tabular}{crllllll}
\multicolumn{5}{c}{ MACHOS } & & HEMBRAS \\
EDAD & N. $^{\circ}$ & MEDIA & DE & N & MEDIA & DE \\
1 & 104 & 146,79 & 23,98 & 57 & 137,30 & 28,37 \\
2 & 179 & 233,95 & 42,41 & 140 & 242,06 & 47,20 \\
3 & 176 & 323,200 & 40,72 & 131 & 343,48 & 46,94 \\
4 & 128 & 406,56 & 50,60 & 81 & 440,11 & 50,57 \\
5 & 102 & 494,28 & 41,68 & 98 & 536,83 & 59,29 \\
6 & 64 & 562,55 & 56,62 & 62 & 622,26 & 75,96 \\
7 & 45 & 616,33 & 59,03 & 61 & 696,71 & 59,96 \\
8 & 18 & 687,31 & 56,32 & 23 & 756,06 & 55,45 \\
9 & 6 & 708,89 & 72,69 & 20 & 838,73 & 94,88 \\
10 & & & & 4 & 893,67 & 92,09 \\
11 & & & & 1 & 881,55 & \\
12 & & & & 1 & 978,49 & \\
13 & & & & 1 & 972,03 &
\end{tabular}

Tabla 1. Valor de la longitud media (MEDIA. mm) y desviación estándar (DE) para cada clasc de edad y sexo Table 1 Valtues of mean length and standar deviation for each agt! class and sex 


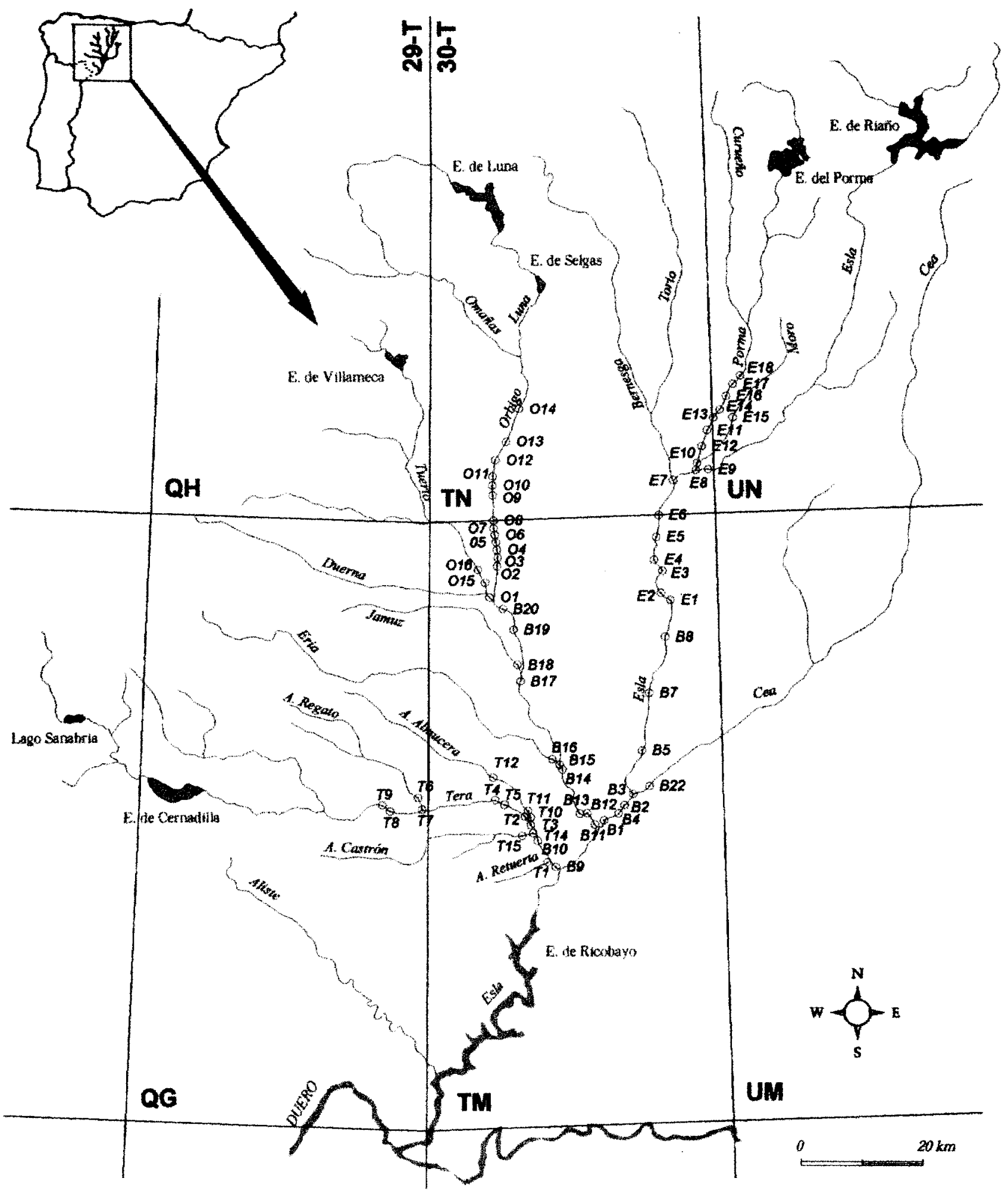

Figura 1. Localización de los puntos de muestreo: E/Esla, O (Orbigo), T (Tera) y B (Zona de barbo). Figure 1. Sampling point distribution. 
Además se han calculado el coeficiente de condición o factor de condición de Fulton y el coeficiente de condición relativa descrito por Le Cren (WEATHERLEY, 1972).

\section{RESULTADOS}

El mejor ajuste lineal $\left(r^{2}=0,95\right)$ se obtuvo entre el radio antcrior de la escama y la longitud total del ejemplar por lo que se utilizó este para estimar la longitud en el momento de formación del último annulus.

La elevada correlación exponencial entre el radio posterior de la escama y la longitud de los ejemplares revela un crecimiento alométrico respecto dc la longitud, mientras que aquella entre el radio anterior y la longitud de los individuos aporta una mayor correlación lineal $\left(r^{2}=0,95\right)$. Por ello se eligió este último para realizar la regresión que permitiera posteriormente efectuar la estimación de las longitudes pretéritas.

El análisis de covarianza de la longitud total (LT) en función del radio anterior de la escama (Ra) y el sexo mostró diferencias entre sexos $(\mathrm{p}<0,0001)$ siendo las ecuaciones propuestas $\mathrm{LT}=$ $7,94+107,72 \mathrm{Ra}(\mathrm{r}=0,969, \mathrm{~N}=713)$ para las hembras y $\mathrm{LT}=$ $39,53+91,61 \mathrm{Ra}(\mathrm{r}=0,974, \mathrm{~N}=866)$ para machos.

Los valores medios de la longitud total para cada clase de edad y sexo se muestran en la Tabla 1. El análisis de varianza realizado muestra que las longitudes medias calculadas para cada edad comienzan a diferenciarse significativamente entre sexos $(p<0.01)$ a partir del tercer año.

Las longitudes medias de cada clase de edad y sus respectivas tasas de crecimiento anual para machos y hembras por separado se muestran en la Figura 2. En el primer año los machos alcanzan una talla superior a la de hembras, a partir del segundo año las tallas medias de cada clase de edad siempre son superiores en las hembras. El descenso en la tasa de crecimiento es mínimo desde el segundo al quinto año, a partir de este momento el efecto se hace más patente, con oscilaciones en las edades más elevadas. Esta variación puede estar ocasionada por la amplia desviación de las longitudes individuales a la media de las clases de edad más alta, cuando, además, el número de muestras es reducido. Es un hecho normal que cuando aumenta la edad las variaciones individuales en el crecimiento se acentúa Pese a todo el incremento anual en longitud se mantiene elevado para todas las clases de edad.

Se encontraron diferencias significativas entre las ecuaciones dc crecimiento calculadas para los dos sexos, por ello las constantes de la ecuación de Von Bertalanffy, que describe el crecimiento teórico en longitud, se han calculado por separado para cada sexo obteniendo los valores relacionados en la Tabla 2.

\section{Relación peso-longitud y coeficientes de condición}

Los resultados del análisis de covarianza realizado para detectar la influencia del sexo sobre el crecimiento en peso muestran diferencias significativas $(\mathrm{p}<0,0001)$. La longitud, que fue considerada como covariablc, explica la mayor parte del crecimiento en peso. No obstante las diferencias entre los sexos son significativas tanto para los puntos de corte (sexo) como para las pendientes ( $\log \mathrm{LT} *$ sexo). Las rectas estimadas para cada sexo son $\log \mathrm{W}=-2,316+3,111 \mathrm{Log} \mathrm{LT}$ para hembras y $\log$ $\mathrm{W}=-2,181+3,015 \log \mathrm{LT}$ en los machos

\begin{tabular}{|c|c|c|c|c|}
\hline & Media & Desv estándar & Límites de & confianza \\
\hline \multicolumn{5}{|c|}{ MACHOS } \\
\hline $\mathrm{L}_{\infty}$ & 1438,39 & 129,36 & 1148,93 & 1691,85 \\
\hline $\mathrm{K}$ & 0,077 & 0,009 & 0,057 & 0,095 \\
\hline$t_{0}$ & $-0,412$ & 0,074 & $-0,557$ & $-0,266$ \\
\hline \multicolumn{5}{|c|}{ HEMBRAS } \\
\hline $\mathrm{L}_{\infty}$ & 1583,52 & 114,38 & 1359,32 & 1807,71 \\
\hline $\mathrm{k}$ & 0,081 & 0,008 & 0,064 & 0,098 \\
\hline $\mathbf{t}_{0}$ & $-0,066$ & 0,073 & $-0,213$ & $-0,073$ \\
\hline
\end{tabular}


Tabla 3. Valores de las constantes de la ecuación de regresión Log W=Log a + b Log LT, separadaniente para cada sexo y mes.

Table 3. Values of mean length and standar deviation for each age age class and

\section{MACHOS}

\section{$\log \mathbf{a} \quad b$}

$\begin{array}{lllll}\text { Enero } & -3,375 & 3,153 & -2,760 & 3.413 \\ \text { Febrero } & -2,104 & 2,986 & -2,357 & 3,152 \\ \text { Marzo } & -2,241 & 3,042 & -2,354 & 3,123 \\ \text { Abril } & -2,122 & 2,973 & -2,383 & 3,084 \\ \text { Mayo } & -2,149 & 3,003 & -2,243 & 3,085 \\ \text { Junio } & -2,234 & 3,055 & -2,249 & 3,079 \\ \text { Julio } & -2,233 & 3,093 & -2,233 & 3,090 \\ \text { Agosto } & -2,082 & 2,983 & -2,102 & 2,994 \\ \text { Septiembre } & -2,378 & 3,173 & -2,334 & 3.136 \\ \text { Octubre } & -2,426 & 3,181 & -2,440 & 3,190 \\ \text { Noviembre } & -2,254 & 3,082 & -2,347 & 3,148 \\ \text { Diciembre } & -2.419 & 3,163 & -2,649 & 3,328\end{array}$

Estas diferencias se explican porque las gónadas en las hembras alcanzan un valor medio de hasta el $20 \%$ del peso corporal en el mes de febrero, período previo a la puesta, mientras se mantiene en valores por debajo del $3 \%$ desde que hasta el mes de agosto que coincide con un descenso en la tasa de ingesta.

La variación del índice en función de las tallas de los ejemplares, separadamente para machos y hembras, se pueden observar en la Figura 4 (B). En los dos casos se advierte una tendencia a aumentar a la vez que lo hace la talla. En las hembras no deja de estar relacionado con el incremento en peso de las gónadas. Para éstas la relación entre el valor del coeficiente de condición y la longitud ha mostrado uria relación positiva y significativa $(\mathrm{p}<0,01)$, por lo que puede establecerse la ecuación de regresión: $\mathrm{K}=0,6514+0,0002$ LT .

Los valores medios del índice y su desviación estándar, para cada mes y talla considerados se incluyen en la Tabla 4.

Calculado el coeficiente de condición para aquellos ejemplares que no tenían el suficiente desarrollo gonadal como para ser sexados (indiferenciados) se obtuvo un valor medio de $0,66( \pm 0,2)$, inferior a los valores mostrados por los adultos.

El coeficiente de condición relativa fue calculado para cada individuo y expresado por meses, pues la relación longitud -peso mostró diferencias. Para los diferentes meses y para las diferentes clases de talla establecidas se obtienen valores próximos a la unidad. Los valores medios y las desviaciones, para cada caso se incluyen en la Tabla 5.

la freza ha concluido (mayo) hasta que vuelve a incrementarse el volumen ovárico (octubre) (DOMÍNGUEZ, 1996). Por su parte los machos no han mostrado medias superiores al $3 \%$.

Para constatar si las diferencias detectadas se mantienen a lo largo del año se realizó un nuevo análisis de covarianza incluyendo el factor mes. Los resultados evidencian diferencias significativas $(p<0,0001)$ tanto para la variación de los puntos de corte (sexo*mes) como para la de las pendientes ( $\left.\log \mathrm{LT}^{*}{ }_{\mathrm{sexO}} * \mathrm{mes}\right)$ por lo que se procedió a calcular las constantes de la ecuación, separadamente para cada sexo y mes (Tabla 3 ).

La Figura 3 muestra la variación, para los distintos meses y sexos, de la pendiente estimada y en la Figura 4 se muestra la variación mensual de la media del coeficiente de condición. En líneas generales son inferiores los correspondientes a machos y la distancia entre las medias de cada mes se acentúa durante el período de maduración gonadal. A partir del momento en que ha concluido la freza (abril) se observa una recuperación del coeficiente de condición, que podría estar relacionada con la mayor ingesta de alimento que se produce durante esos meses (PENA et al., 1987)



Figura 2. Longitudes medias de cada clase de edad ( $\mathrm{H}=$ hembras, $M=$ machos) y sus respectivos incrementos de longitud (Incr $\mathrm{h}=$ incremento hembras, $\operatorname{lncr} \mathrm{ni}=$ incremento machos) Figure 2. Means lengths (in $\mathrm{mm}$ ) in each age group ( $\mathrm{H}=$ male). Length annual increase for both sex. 



Figura 3. Variación del coeficiente de alometría (b), pendiente de la recta de regresión de la ecuación $\log W=\mathrm{Log}$ a + b Lo: I.T, eii los diferentes meses del año, separadainente para machos y hembras.

Figure 3. Length-weight regression coefficient (b) calculated monthly nnd separately for both sex 


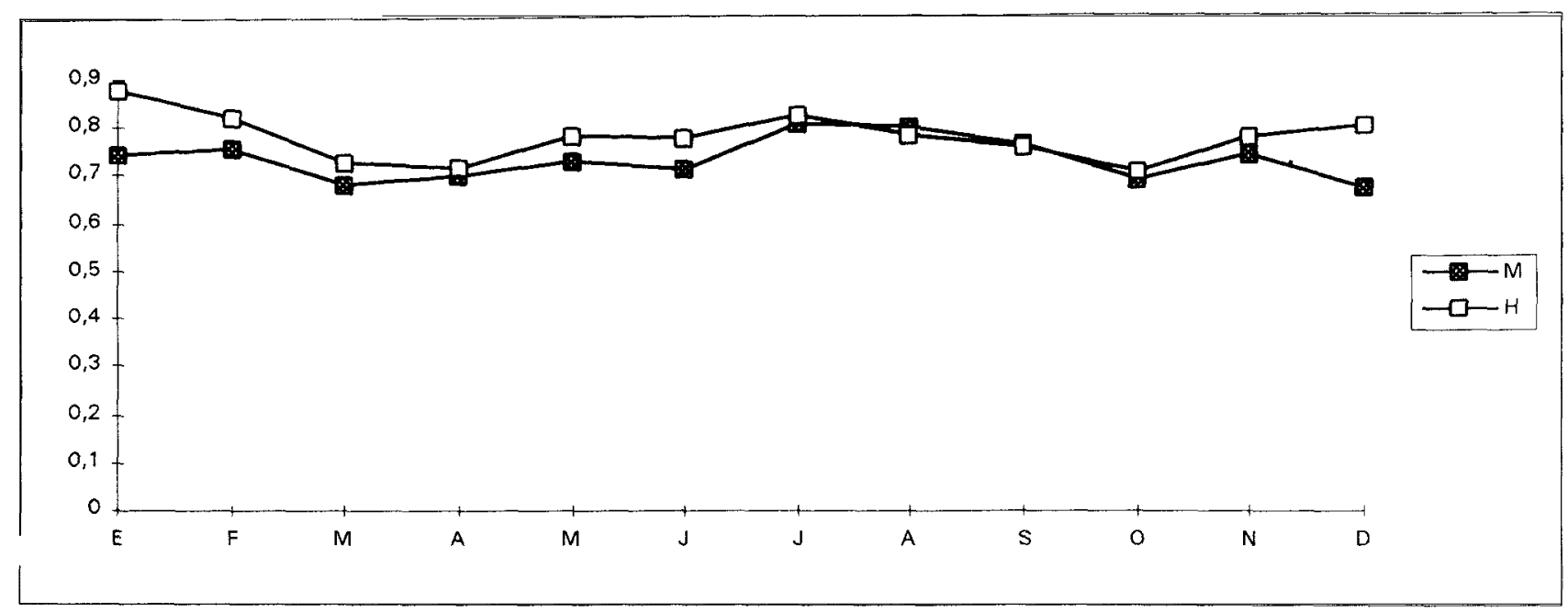

A

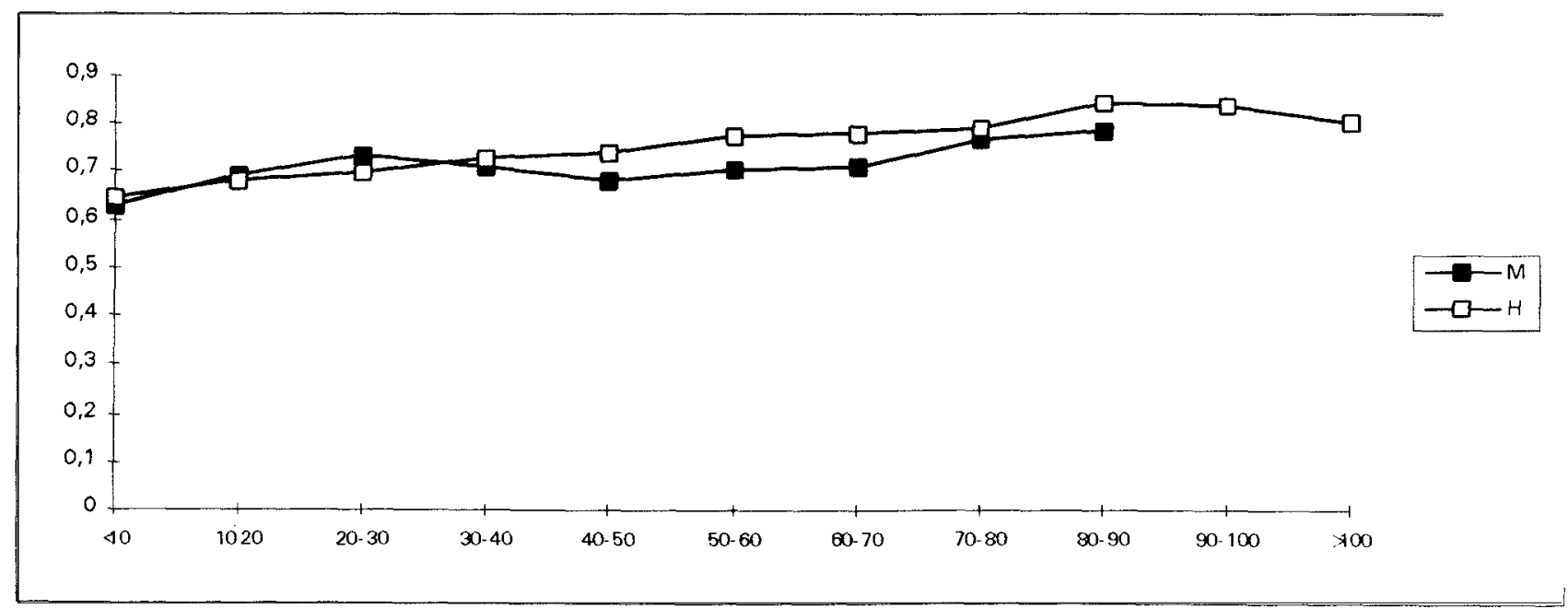

$\mathbf{B}$

Figura 4. A Variación mensual de la media del coeficiente de condición separadamente para cada sexo. B. Variación del coeficiente de condición en función de la talla. Se representan los valores medias para cada clase de talla considerada y sexo. ( $M=$ machos, $H=h e m b r a s)$. Figure 4. Coefficient condition average. A Monthly variation for both sex. B. Variation on length. ( $M=$ males, $H=$ females). 


\section{DISCUSIÓN}

\section{Crecimiento. Longitud de cada clase de edad}

De forma general las hembras alcanzan mayor longitud que los machos para una misma clase de edad. Las diferencias son mínimas en las primeras edades y se acentúan con la edad. Así ha sido registrado por numerosos autores.

Los resultados obtenidos en el presente estudio muestran que, en el primer año, la talla inedia de los machos es ligeramente superior a la de las hembras, mientras para las siguientes clases de edad fueron siempre mayores los valores medios alcanzados por éstas, hecho coincidente con lo afirmado por CALDERONI (1965), WOLFERT \& MILLER (1978), CRAIG \& SMILEY (1986) y WRIGHT (1990).

Las diferencias de las medias de longitud para cada clase de edad son significativas a partir del tercer año. Las situaciones que revelan otros trabajos, a este respecto, son diversas. Así TREASURER et al. (1992) no encuentran diferencias entre sexos, en un lago, mientras en otro comienzan a diferenciarse a partir del tercer año. ALESSIO (1983), concluye que los machos superan la longitud alcanzada por las hembras hasta los cinco años, momento en que la situación se invierte.

En nuestro estudio los individuos de uno y dos años de edad presentan longitudes medias inferiores a las obtenidas por otros autores. Sin embargo se registra un crecimiento superior en las edades siguientes, lo que hace que, en ocasiones, no solo alcancen sino que sobrepasen los valores de referencia.

Las tallas alcanzadas por los lucios de la cuenca del Esla son menores que las registradas por otros autores (Ver Tablas 6, 7 y 8). Solo superan a las descritas por MUNRO (1957) para un lago escocés y son muy próximas a las descritas por ALESSIO (1975 b) para la cucnca alta del Po (Italia) y CALDERONI (1 965) en el lago Trasimeno (Italia).

Si se analizan por clases de edad, a partir de los tres años los lucios de la cucnca del Esla recuperan posiciones respecto a los datos aportados por otros siete autores: SNOW \& BEARD

Tabla 4. Valores medios del coeficiente dc condición y desviación estándar (DE para los diferente? meses y tallas consideradas.

Table 4. Means values for condition coefficient and S.D. for each month and size class.

MACHOS

\begin{tabular}{|c|c|}
\hline MESES & $\mathbf{N}^{\prime \prime}$ \\
\hline Enero & 49 \\
\hline Febrero & 130 \\
\hline Marzo & 1.019 \\
\hline Abril & 468 \\
\hline Mayo & 50 \\
\hline Junio & 93 \\
\hline Julio & 35 \\
\hline Agosto & 125 \\
\hline Septiembre & 169 \\
\hline Octubre & 50 \\
\hline Noviembre & 43 \\
\hline Diciembre & 54 \\
\hline $\begin{array}{l}\text { TALLAS } \\
(\mathrm{mm})\end{array}$ & N" \\
\hline$<10$ & 9 \\
\hline $10-20$ & 283 \\
\hline $20-30$ & 518 \\
\hline $30-40$ & 461 \\
\hline $40-50$ & 377 \\
\hline $50-60$ & 348 \\
\hline $60-70$ & 229 \\
\hline $70-80$ & 54 \\
\hline $80-90$ & 5 \\
\hline $90-100$ & \\
\hline$>100$ & \\
\hline
\end{tabular}

\section{HEMBRAS}

$\begin{array}{ll}\text { Media } & \text { DE } \\ & \\ 0,774 & 0,107 \\ 0,755 & 0,136 \\ 0,681 & 0,117 \\ 0,698 & 0,151 \\ 0,726 & 0,110 \\ 0,709 & 0,112 \\ 0,799 & 0,119 \\ 0,794 & 0,017 \\ 0,758 & 0,167 \\ 0,688 & 0,106 \\ 0,739 & 0,082 \\ 0,669 & 0,094 \\ & \\ \text { Media } & \text { DE } \\ & \\ 0,629 & 0,0814 \\ 0,691 & 0,1857 \\ 0,733 & 0,0185 \\ 0,707 & 0,0993 \\ 0,679 & 0,1088 \\ 0,700 & 0,0964 \\ 0,709 & 0,0879 \\ 0,767 & 0,0977 \\ 0.783 & 0,1231 \\ & \\ \end{array}$

$\begin{array}{rll}\mathbf{N}^{\mathbf{o}} & \text { Media } & \text { DE } \\ & & \\ 52 & 0,877 & 0,161 \\ 207 & 0,817 & 0,145 \\ 1.011 & 0,723 & 0,163 \\ 284 & 0,715 & 0,133 \\ 35 & 0,778 & 0,123 \\ 72 & 0,772 & 0,101 \\ 41 & 0,821 & 0,143 \\ 79 & 0,780 & 0,090 \\ 158 & 0,752 & 0,084 \\ 52 & 0,702 & 0,117 \\ 54 & 0,771 & 0,098 \\ 40 & 0,798 & 0,159 \\ & & \\ \mathbf{N}^{\circ} & \text { Media } & \mathbf{D E} \\ & & \\ 4 & 0,645 & 0,0537 \\ 171 & 0,682 & 0,2118 \\ 324 & 0,699 & 0,1418 \\ 326 & 0,728 & 0,1186 \\ 277 & 0,738 & 0,1304 \\ 380 & .0,770 & 0,1401 \\ 318 & 0,780 & 0,1509 \\ 180 & 0,791 & 0,1492 \\ 72 & 0,840 & 0,1447 \\ 30 & 0,838 & 0,1583 \\ 3 & 0,803 & 0,0670\end{array}$


Tabla 5. Valores medios y desviaciones estándar del coeficiente de condición relativa para los diferentes meses y clases de talla consideradas.

Table 5. Means values and S.D. of relative condition coefficient for each month and size class.

MACHOS

$\begin{array}{lrrr}\text { MESES } & \text { N" } & \text { Media } & \text { DE } \\ \text { Enero } & 49 & 1,008 & 0,14 \\ \text { Febrero } & 130 & 1,013 & 0,18 \\ \text { Marzo } & 1.019 & 1,013 & 0,18 \\ \text { Abril } & 468 & 1,017 & 0,21 \\ \text { Mayo } & 50 & 1,013 & 0,15 \\ \text { Junio } & 93 & 1,011 & 0,16 \\ \text { Julio } & 35 & 1,009 & 0,14 \\ \text { Agosto } & 125 & 1,013 & 0,21 \\ \text { Septiembre } & 169 & 1,014 & 0,22 \\ \text { Octubre } & 50 & 1,004 & 0,10 \\ \text { Noviembre } & 43 & 1,005 & 0,10 \\ \text { Diciembre } & 54 & 1,006 & 0,11\end{array}$

TALLAS

$<10$

$10-20$

20-30

$30-40$

$40-50$

$50-60$

$60-70$

$70-80$

$80-90$

$90-100$

$>100$

$\begin{array}{rr}\mathbf{N}^{\circ} & \text { Media } \\ & \\ 9 & 0,955 \\ 283 & 1,006 \\ 518 & 1,042 \\ 461 & 1,017 \\ 377 & 0,981 \\ 348 & 1,004 \\ 229 & 1,013 \\ 54 & 1,039 \\ 5 & 1,080\end{array}$

\section{HEMBRAS}

$\begin{array}{rll}\mathbf{N}^{\mathbf{}} & \text { Media } & \text { DE } \\ 52 & 1,007 & 0,12 \\ 207 & 1,015 & 0,17 \\ 1.011 & 1,023 & 0,23 \\ 284 & 1,016 & 0,18 \\ 35 & 1,011 & 0,15 \\ 72 & 1,007 & 0,12 \\ 41 & 1,011 & 0,17 \\ 79 & 1,006 & 0,12 \\ 158 & 1,004 & 0,09 \\ 52 & 1,004 & 0,09 \\ 54 & 1,003 & 0,09 \\ 40 & 1,008 & 0,13 \\ & & \\ \mathbf{N} " & \text { Media } & \mathbf{D E} \\ & & \\ 4 & 0,979 & 0,09 \\ 171 & 1,034 & 0,32 \\ 324 & 1,006 & 0,20 \\ 326 & 1,010 & 0,16 \\ 277 & 1,011 & 0,17 \\ 380 & 1,025 & 0,17 \\ 318 & 1,015 & 0,18 \\ 180 & 1,001 & 0,17 \\ 72 & 1,042 & 0,17 \\ 30 & 1,015 & 0,19 \\ 3 & 0,959 & 0,03\end{array}$

No obstante el crecimiento del lucio muestra una enorme variabilidad, alcanzando mayores tallas, en general, en lagos y embalses RRACKEN (1973). FROST \& KIPLING (1967) encuentran modificaciones en el crecimiento cuando la población ha sido sometida a sobrepesca que afectan fundamentalmente a los grandes ejemplares. Como consecuencia se detecta un increinento del crecimiento, que es superior en los de mayor cdad.

El primer trabajo sobre lucio en nuestro país (GUTIÉRREZCALDERON, 1951), ofrece las longitudes máximas alcanzadas por ejemplares de dos meses que llegaron a los 12 cin de longitud. Como contraste en la cuenca del Esla ejemplares de dos meses miden solo 5,5 cm (AGUNDEZ, 1986). En un trabajo posterior de GUTIÉRREZ-CALDERON (1954) ofrece el dato de $45 \mathrm{~cm}$ al año de cdad en estanque, 70 $\mathrm{cm}$ cuando tienen dos años y $90 \mathrm{~cm}$ a los cuatro. También GUTIÉRREZ-CALDERON (1969) indica las longitudes que han alcanzado en aguas libres. en el río Tajo, 53 y $54 \mathrm{~cm}$ a 10 s 7 y 11 meses, $65 \mathrm{~cm}$ con 2 años, 87 y $97 \mathrm{~cm}$ a los tres años y 95 a los cuatro. Todos ellos son registros aislados. 


\begin{tabular}{|c|c|c|c|c|c|c|c|c|c|c|c|c|c|c|}
\hline AUTOR & ZONA DE ESTUDIO & LTI & LT2 & LT3 & LT4 & LT5 & LT6 & LT7 & LT8 & LTy & LTIO & LTII & LT12 & LT13 \\
\hline Datos del presente estudio & Cuenca del Esla (España) & & & & & & & & & & & & & \\
\hline Machos & & 14.6 & 23,4 & 32,3 & 40,6 & 49,4 & 56,2 & 61,6 & 68,7 & 70,8 & & & & \\
\hline Hembras & & 13,7 & $2+.2$ & 34,3 & 44 & 53.6 & 62,2 & 69.6 & 75.6 & 83.8 & 89.3 & 88,1 & 97.8 & 97,2 \\
\hline Munro (1957) & Lago de Escocia & & & & & & & & & & & & & \\
\hline Machos & & 7,2 & 18,5 & 29,1 & 36,3 & 41,6 & 46,4 & 49,9 & $\cdot 53,2$ & & & & & \\
\hline Hembras & & 8,2 & 19,3 & 31.1 & 40.3 & 47,4 & 53,6 & 57,4 & 63,4 & 70 & 87 & 90 & 93 & \\
\hline Buss \& Miller (1961) & Lagos de Pennsylvania & 27 & 48 & 58 & 66 & 73,5 & 85 & & & & & & & \\
\hline Snow \& Beard (1972) & Buck Lake. Wisconsin & 20.5 & 32,3 & 38,5 & 43,3 & 47,5 & 51,7 & 55 & 67.3 & 74 & & & & \\
\hline Snow (1974) & Embalse de Wisconsin & & & & & & & & & & & & & \\
\hline Nativos & & 39.6 & 45,7 & 55,6 & 60,2 & 66 & & & & & & & & \\
\hline Repoblados & & 26.9 & 37,1 & 49,3 & 56,9 & 64,8 & & & & & & & & \\
\hline Priegel \& Krohn (1975) & Lagos de Wisconsin & & & & & & & & & & & & & \\
\hline Machos & & 24,9 & 43,2 & $5 \mathrm{I}, 8$ & 56,4 & 59,7 & 62,5 & 65,5 & 68.1 & 70,9 & 72,9 & & & \\
\hline Hembras & & 26,2 & 47.8 & 58,4 & 66 & 69,8 & 73,4 & 79,2 & 85 & 90,1 & & & & \\
\hline Alessio (1975) & Cuenca del Po. Italia & & & & & & & & & & & & & \\
\hline Lomellina: Machos & & 14,7 & 26,6 & 35,2 & 43,9 & 50,5 & 61,9 & 67,9 & 76 & & & & & \\
\hline Hembras & & 15,5 & 27,8 & 36,8 & 44,9 & 52,6 & 59,9 & 65,5 & & & & & & \\
\hline S. Gaetano: Machos & & 19,8 & 31,8 & 40,7 & 46,4 & & & & & & & & & \\
\hline Hembras & & 20,1 & 33,2 & 44,3 & 51,8 & 57,5 & & & & & & & & \\
\hline Paragamian (1976) & Río Plover. Wisconsin & 25 & 43,8 & 58,6 & 68,1 & & & & & & & & & \\
\hline Kempinger \& Carline (1978) & Lago Escanaba. Wisconsin & 36,8 & 48,8 & 53,8 & 60,7 & 66 & 69,6 & 75,9 & & & & & & \\
\hline Wolfert \& Miller (1978) & Lago Ontario & & & & & & & & & & & & & \\
\hline Machos & & 24,6 & 49,3 & 61,2 & 66,5 & 69,3 & 70,5 & 72,6 & 70,7 & & & & & \\
\hline Hembras & & 24 & 51,5 & 67,3 & 73 & 76,7 & 78,6 & 77,1 & 72,9 & & & & & \\
\hline Dauba (1981) & Embalses de Bretaña & & & & & & & & & & & & & \\
\hline Chastang & & 32 & 50 & 62,2 & 67,5 & 69,2 & & & & & & & & \\
\hline Luzecti & & 22,6 & 32,9 & 44,4 & 53 & 60 & 63 & 69,7 & & & & & & \\
\hline Boet \& Le Louarn (1985) & Lagos y embalses de Bretaña & 23,3 & 35,6 & 43,4 & 54,7 & 61,7 & & & & & & & & \\
\hline Neumann et al. (1993) & Lago de Dakota del Sur & & & & & & & & & & & & & \\
\hline Machos & & 45,9 & 49,8 & 55,2 & 61,1 & 69,5 & & & & & & & & \\
\hline Hembras & & 46,5 & 56,5 & 65 & 70,1 & 81,2 & 85,3 & 96 & 98,7 & & & & & \\
\hline
\end{tabular}


Estos resultados son propios dc unas condiciones puntuales y de cursos de agua que ofrecen presas ícticas que posibilitaron tales desarrollos. MARTINEZ (1985) describe para 1970, cn el einbalse de Santillana tallas de 38, 47, 58 y $61 \mathrm{~cm}$ respectivamente para las edades de 1 a 4 años, claramente superiores a las que se aportan en nuestro estudio.

Es de destacar la similaridad mostrada entre nuestros datos y los resultados obtenidos en Italia por CALDERONI (1965) y ALESSIO (1975 b). Los datos de MUNRO (1957) son de una población de lucios de un lago en el que la trucha desapareció dcspues de la introducción del lucio. El grado dc canibalismo y la elevada competencia intraespecífica se traduce en un menor crecimiento.

En cuanto a la longitud teórica máxima $\left(\mathrm{L}_{\approx}\right)$, los elcvados valores obtenidos son reflejo del crecimiento sostenido a lo largo de las clases de edad. Para hembras ha sido estimada en $158 \mathrm{~cm}$ y para machos en 144 (LT). A la vista de cstos resultados puede advertirse que las asíntotas calculadas para las curvas de crecimiento son, en términos generales. más elevadas que las referidas por otros autores. Así únicamente son superiores las longitudes aportadas por SCHINDLER (1975), muy próximos a los valores hallados por HEALY (1956) y NEUMANN et al. (1994) y muy por debajo los de FROST \& KIPLING (1967). MANN (1976), CRAIG \& SMILEY (1986), TREASURER et al. (1992) y WRIGHT (1990).

También cstos cálculos distan de las tallas de los lucios más grandes capturados por nosotros. si bien REGUERAS (1995) en un registro dc capturas de pescadores profesionales en el río Esla, cita dos ejemplares de 20 y $26 \mathrm{Kg}$. A partir de la relación longitud - peso aquí elaborada, el primero de ellos tendría una Iongitud de $141 \mathrm{~cm}$ y el ejemplar de $26 \mathrm{Kg}$ podría medir 154 cin. Paralelamente se afirma que han sido capturados lucios de hasta $150 \mathrm{~cm}$ pero esto debe tomarse con las debidas reservas hasta ser corroborados convenicntemente.
Las constantes de las ecuaciones de crecimiento propuestas (k) son notablemente más bajas que las obtenidas por otros autores, lo que indica que la longitud de los lucios de la cuenca del Esla se aproxima muy lentamente a la asíntota de la curva.

Los valores obtenidos para $\mathrm{k}$ han resultado ser 0,077 para los machos y 0,081 para las hembras. Los datos más bajos referidos en otros trabajos han sido los de CRAIG \& SMILEY (1986) que obtiene valores de 0.16 para inachos y 0,13 en hembras y WRIGHT (1990) que estima 0,21 en machos y 0,12 en hembras. Mayores serían los de FROST \& KIPLING (1967) y MANN (1976). En todos los casos las longitudes de las primeras clases de edad son muy superiores a las calculadas para la cuenca del Esla

Por lo que se refiere a la longevidad, FROST \& KIPLING (1967) inencionan una heinbra de 17 años y dos machos dc 16. SNOW \& BEARD (1972) encuentran el ejemplar de mayor edad con 12 años, en el extremo opuesto BRACKEN (1973) refiere como rara vez encuentra ejemplares de más de 4 años. MANN (1976) registra en el río Stour hembras de 9 años y machos con 10, mientras en el Frome las hcrnbras más longevas alcanzaron los 12 y los machos 5. RAAT (1988) considera que las hembras viven más años, 12 ó 13 como máxiino, pero los machos alcanzarían su máximo en 5 ó 6 . WRIGHT (1990) presenta hembras de 13 años y machos de hasta 9. Idénticos a estos últimos datos son los resultados del presente estudio; la mayor edad alcanzada fue de 13 años por las hembras y 9 por machos.

Relación peso-longitud y coeficientes de condición.

Para comparar los valores del coeficieiite de alometría ( b en la ecuación que relaciona ${ }^{\mathrm{el}}$ peso y la longitud) calcul ados en el presente estudio con los aportados por otros autores se ha tenido en cuenta el período del año y el sexo de los cjemplares, cuando cstos datos son señalados cn los trabaios referidos. También se ha considerado la longitud utilizada.

Tabla 7. Comparación de las longitudes medias obtenidas en el presente estudio con los resultados de los autores relacionados ( LS= Longitud standard eri cm) Table 7. Mean length (Standard Length) values for each age-class of this study compared with results of others authors.

\begin{tabular}{|c|c|c|c|c|c|c|c|c|c|c|c|c|c|c|}
\hline AUTOR & ZONA DE ESTUDIO & LSI & $\mathrm{LS} 2$ & I.S3 & LS4 & LS5 & LS6 & LS7 & LSY & LS9 & LSIO & LSII & LS12 & LS13 \\
\hline Datos del presente estudio & Cucnca del Esla. (Espaniáa) & & & & & & & & & & & & & \\
\hline Maclios & & 12.5 & 20.4 & 28,5 & 36,1 & 44 & 50,2 & 55.1 & 61.5 & 63,4 & & & & \\
\hline Hembras & & 11.7 & 21,2 & 30.3 & 30 & 47,9 & 55,6 & 62,3 & 67.7 & 75.2 & 80.2 & 79.1 & 87,9 & 87,3 \\
\hline Calderoni (1965) & Lago de Italia & & & & & & & & & & & & & \\
\hline Maclios & & 14.7 & 20.8 & 30,3 & 78 & 42,6 & 42,1 & & & & & & & \\
\hline Hembras & & 14.2 & 23 & 32.7 & 40,6 & 48.6 & 56,8 & 67 & 70.9 & 72,9 & & & & \\
\hline Vostradowsk! (1977) & Embalse Checoslovaquia & & & & & & & & & & & & & \\
\hline Machos & & 25,9 & 39 & 47.1 & 53 & 59,8 & 63,5 & & & & & & & \\
\hline Hembras & & 27.9 & 41,1 & 51,6 & 61.8 & 72.1 & 83 & 91,2 & 93 & & & & & \\
\hline Penczak et al. (1986) & Rios none de Polonia & 19.5 & 22.8 & 27.7 & 31.7 & 37,6 & 40.4 & 44.7 & 47.2 & 50.8 & 53.5 & 55.5 & & \\
\hline Pivnika (1991) & Embalse Checoslovaquia & 18 & 30 & 42 & 52 & 61 & 67 & 71 & 76 & & & & & \\
\hline Cerni (1992) & Danubio, Checoslovaquia & 16.3 & 28 & 35,3 & 41.5 & 48,4 & 59.9 & 67.7 & & & & & & \\
\hline
\end{tabular}




\begin{tabular}{|c|c|c|c|c|c|c|c|c|c|c|c|c|c|c|c|c|}
\hline $\begin{array}{l}\text { AUTOR } \\
\text { Datos del presente estudio }\end{array}$ & $\begin{array}{l}\text { ZONA DE ESTUDIO } \\
\text { Cuenca del Esla (España) }\end{array}$ & LTal & LTa2 & LTa3 & LTa4 & LTa5 & LTa6 & LTa7 & LTa8 & LTa9 & LTalo & LTall & LTal2 & LTa13 & LTalt & LTa15 \\
\hline Machos & & 13.7 & 22.1 & 30.7 & 38.7 & 47.2 & 53.7 & 58,9 & 64.7 & 67.8 & & & & & & \\
\hline Hembras & & 12,8 & 22,9 & 32,6 & 41,9 & 51,2 & 59,4 & 66,6 & 72,3 & 80.3 & 85,5 & 84,4 & 93.7 & 93,1 & & \\
\hline Healy (1956) & Lagos de Irlanda & & & & & & & & & & & & & & & \\
\hline Lough Rca & & 26 & 45 & 60 & 72 & 84 & 94 & 103 & 104 & & & & & & & \\
\hline Lough Glore & & 22 & 36 & 49 & 61 & 74 & 86 & 95 & 106 & .112 & & & & & & \\
\hline Barnagrow Lake & & 18 & 29 & 40 & 50 & 58 & 64 & 71 & 86,8 & & & & & & & \\
\hline Frost \& Kipling (1959) & Lago Windermere U.K & & & & & & & & & & & & & & & \\
\hline Machos & & 23,1 & 40 & 52,1 & 59,1 & 63,3 & 65,4 & 66 & 68 & 68.4 & 70,4 & 72 & 71,5 & 71,3 & 72,5 & 73,5 \\
\hline Hembras & & 22.7 & 39,9 & 55,3 & 64,4 & 69.8 & 75.7 & 79.7 & 84,4 & 87,8 & 90,4 & 92,8 & 96,3 & 101,7 & 102,5 & \\
\hline Frost \& Kipling (1967) & Lago Windermere U.K & & & & & & & & & & & & & & & \\
\hline datos 1930-38 Machos & & 21 & 37 & 48 & 54 & 59 & 62 & 64 & & & & & & & & \\
\hline Hembras & & 22 & 38 & 52 & 61 & 69 & 75 & 79 & & & & & & & & \\
\hline datos 1951-55: Machos & & 22 & 38 & 52 & 61 & 69 & 75 & 79 & & & & & & & & \\
\hline Hembras & & 23 & 39 & 52 & 59 & 64 & 68 & 71 & & & & & & & & \\
\hline Bracken \& Champ (1971) & Lagos de Irlanda & & & & & & & & & & & & & & & \\
\hline Lough Corrib: Machos & & 25,8 & 44.5 & 58,8 & 68.2 & 73,8 & 75,4 & & & & & & & & & \\
\hline Hembras & & 26,5 & 48 & 63 & 76,9 & 85,6 & 93,6 & 102,6 & & & & & & & & \\
\hline Lough Sillan: Machos & & 25,5 & 38,3 & 49.3 & 57,1 & 62,6 & 68 & 72,3 & & & & & & & & \\
\hline Hembras & & 25,6 & 42,3 & 54,6 & 64,9 & 74,2 & 86.4 & & & & & & & & & \\
\hline Mann (1976) & Ríos de Inglaterra & & & & & & & & & & & & & & & \\
\hline Rio Stour: Machos & & 25 & 40,2 & 51,7 & 58,2 & 62,6 & 66,8 & 69,8 & 72 & 75,9 & 77,2 & & & & & \\
\hline Hembras & & 25,3 & 41,3 & 53,5 & 62,4 & 70,2 & 76,2 & 79,4 & 88,4 & 93,6 & & & & & & \\
\hline Rio Frome: Machos & & 20.7 & 37,6 & 50.7 & 64,4 & 73,2 & & & & & & & & & & \\
\hline Hembras & & 22.6 & 36,9 & 52.2 & 63,7 & 73 & 83.7 & 891 & 94.8 & 96.7 & 100,2 & 102.9 & 106 & & & \\
\hline Gee (1978) & Lagunas S.E. Inglaterra & 20 & 30 & 40 & 50 & & & & & & & & & & & \\
\hline Oliva y Naiksatam (1979) & Rio Dunajec. Rep. Checa & 19,8 & 28,9 & 37,9 & 49,4 & 55 & 70,2 & 80 & & & & & & & & \\
\hline Le Louarn \& Bagliniere (1985) & Río Scorf, Francia: curso alto & 17,8 & 30.9 & 36,9 & 38,8 & & & & & & & & & & & \\
\hline & curso bajo & 27,8 & 47 & 60,9 & 68,1 & & & & & & & & & & & \\
\hline Craig \& Smiley (1986) & Lagos Alberta & & & & & & & & & & & & & & & \\
\hline Ethel : Machos & & 22 & 34 & 40 & 45 & 48 & 51 & 53 & 55 & & & & & & & \\
\hline Hembras & & 20 & 37 & 42 & 48 & 51 & 53 & 56 & 59 & & & & & & & \\
\hline Marie: Machos & & 22 & 34 & 43 & 50 & 52 & 55 & 57 & 60 & & & & & & & \\
\hline Hembras & & 22 & 35 & 47 & 53 & 57 & 62 & 65 & 69 & & & & & & & \\
\hline Wolf: Machos & & 21 & 33 & 41 & 45 & 49 & 52 & 55 & 57 & & & & & & & \\
\hline Hembras & & 20 & 33 & 42 & 48 & 52 & 55 & 62 & 64 & & & & & & & \\
\hline Wright (1990) & Lagos U.K & & & & & & & & & & & & & & & \\
\hline Main Lake. Machos & & 25.4 & 32,3 & 43,9 & 48,3 & 56,1 & 60,2 & 64.3 & 65.1 & 71 & & & & & & \\
\hline Hembras & & 23.3 & 37 & 47,9 & 55,6 & 61,6 & 65.3 & 73.2 & 79.3 & 86.8 & 90.2 & 85 & 92,5 & 102 & & \\
\hline St Peters. Machos & & 21.5 & 28.1 & 35 & 43.1 & 45 & 51.2 & 47 & 68 & & & & & & & \\
\hline Hembras & & 24.1 & 28.5 & 35.7 & 43.9 & 47.1 & 50.7 & 57.8 & 66.5 & 84.3 & 94 & 83 & 94 & 96 & & \\
\hline
\end{tabular}


Valores muy próxiinos son los rnericionados por MUNRO (1957), HOLCICK (1968). MANN (1976), LE LOUARN \& BAGLINIERE (1985), PENCZAK et al. (1986) y PROKES (1993). También es inuy coincidente con el valor del coeficiente de la ecuación estándard propuesta por WILLIS (1989) realizada sobre 23 poblaciones de lucio de Estados Unidos y Canadá.

Ligeramente inferior es la constante mostrada por CALDERONI et al. (1980) para los dos sexos. Por el contrario son superiores, en términos generales, los coeficientes calculados por FROST \& KIPLING (1967), ALESSIO (1975 a), PARAGAMIAN (1976), KEMPINGER \& CARLINE (1978), WRIGHT (1990) y PIVNICKA (1991).

Los resultados obtenidos en este trabajo son, para el total de los ejemplares, muy próximos al valor teórico tres, si bien, como ya se ha descrito en el apartado de resultados, las variaciones están muy influenciadas por el estado gonadal. Por ello las diferencias a lo largo del año son más acusadas en las hembras.

El coeficiente de condición es inferior al mostrado por HOLCIK (1968) y superior al referido por BAUCHOT \& RAUCHOT (1978).

La condición relativa. por su parte, se muestra inás constante, en torno al valor 1, tanto para los meses como para las clases de talla consideradas. No ocurre así con los resultados obtenidos por MANN (1976) quien encuentra variaciones de la media a lo largo de los meses del año.

\section{AGRADECIMIENTOS}

A los Servicios de Vida Silvestre de la Junta de Castilla y León dc León y Zamora por las facilidades y la colaboración en la captura de los ejemplares. A R. Aguado por su labor en la preparación y lectura de escamas. A R. Ontañón y al Dr. Luis Calabuig por la ayuda prestada en el tratamiento de los datos.

\section{BIBLIOGRAFÍA}

ACUNDEZ. P. 1986. Estudio dc la reproducción y desarrollo larvario del lucio, Esox lucius L. 1758, en la cuenca del Esla. Memoria de Licenciatura. Universidad de León. 87 PP.

ALESSIO, G. 1975 a. Richerche sulla biologia del luccio, Esox lucius L. (Osteichthyes, Esocidae), in Lomellina Occidentale ed in un "Valle" Veneta. Boll. Pesca Piscic. Idrobiol., 30 (2): 235-256.

ALESSIO, G. 1975 b. Accrescirnento lineare e ponderale del luccio, Esox lucius L. (Osteichthyes, Esocidae), in Lomclliria Occidentale ed in una "Valle" Veneta. Boll. Pesca Piscic. Idrobiol., 30 (2): 257-275.

ALESSIO, G. 1983. Quelques aspects de la biologie el de l'élevage du brochet (Esox lucius L.) en Italie. En: Billard, R. cd. Le brochet: gestion dans le milieu naturel et élevage. INRA Publ., Paris, 283-296.
RAGENAL, T.R. \& F.W. TESCH. 1978. Age and growth. En Bagenal, T.B. ed., Methods for assessment of fish production in fresh waters. IBP Handbook N" 3. Blackwell Scientific Publications. Oxford: 101-136.

BAUCHOT. R \& M.L. BAUCHOT. 1978. Coefficient de condition et indice pondéral chez les Téléodtéens. Cybium. 3 (4): 3-16.

BOET, P. \& H. LE LOUARN. 1985. La croissance du poisson. Techniques d'étude. En: Gerdeaux, D \& R. Billard ed. Gestion piscicole des lacs et retenues artificielles. INRA. Paris, 125-142.

BRACKEN, J.J. 1973. The age and growth of pike Esox lucius from Irish trout rivers. Ir. Fish. Invest. (A Freshwater), 12: 3-15.

CALDERONI, P. 1965. Contnbuto allo studio dell'acrescirnento del luccio (Esox lucius L.) del lago Trasiincno. Riv. Idrobiol., 4: $3-15$.

CALDERONI, P., G. GIOVINAZZO, M. MEARELLI \& L. VOLPI. 1980.Contributo alla conosccnza di Esox lucius L. del lago Trasimeno. Riv. Idrobiol. :347-359.

CRAIG, J.F. \& K, SMILEY. 1986. Wayelle, Stizostedion vitreum, and northern pike, Esox lucius, populations in three Alberta lakes. J. Fish Biol., 29: 67-85.

CHEN, Y., D.A. JACKSON, \& H.H. HARVEY. 1992. A comparison for Von Rertalanffy and polynomial functions in rnodelling fish growth data. Can. J. Fish. Aquat. Sci., 49 (6): 1228-1235.

DAUBA, F. 1981. Etude comparative de la fauna des poissons dans les ecosystemes de deux reservoirs: Luzech (Lot) et Chastang (Dordogne). These. Institut National Polytechnique de Toulouse. $228 \mathrm{pp}$.

DIANA, J.S. 1987. Simulation of mechanisms causing stunting in northern pike populations. Trans. Am. Fish. Soc. 116: 612-617.

DOMINGUEZ, J. 1996. Contribución al conocimiento de la biología del lucio (Esox lucius) cn uri área de reciente colonización: Cuenca del Esla. Tesis Doctoral. Universidad de León.

FROST, W. 1954. The food of pike, Esox lucius L., in Windermere. I. Anim. Ecol., 23: 339-360.

FROST, W. \& C. KIPLING. 1959. The determination of the age and growth of pike (Esox lucius L.) from scales and opercular bones. Journal du Conseil permanent international pour l'exploration de la mer, 24: 314-341.

FROST, W. \& C. KIPLING. 1967. Removal of pike (Esox lucius) from Windcrmere and some of its effect on the population dynamics of that fish. Proceedings of the third British Coarse Fish Conference. Liverpool, 53-56. 
GUTIERREZ-CALDERON, E. 1951. L'acclimatation du brochet en Espagne. Travaux de l'Association Internationale de Limnologie théorique et appliquée, XI: 69-74.

GUTIERREZ-CALDERON, E. 1954. El lucio en España. Caza y Pesca. 140: 490-493.

GUTIERREZ-CALDERON, E. 1969. El lucio (su biología y aprovechamiento). Ministerio de Agricultura. Madrid. 87 pp.

HEALY. A. 1956. Pike (Esox lucius L.) in three Irish Lakes. Sci. Proc. R. Dublin Soc., 27: 51-63.

HOLCIK, J. 1968. Lifc history of the pike Esox lucius Linnaeus, 1758, in thc Klícava reservoir. Véstník Cs. Spol. Zool. 32 (2): 166-180.

KEMPINGER, J.J. \& R.F. CARLINE. 1978. Changes in population density, growth, and harvest of northern pikc in Escanaba lake after iinpleinentation of a 22-inch size limit. Dep. Nat. Res. Madison. Wisconsin. Techn. Bull., N" 104:1-15.

LE LOUARN, H. \& J.L. HAGLINIERE. 1985. Quelques élenients de la biologie du brochet (Esox lucius L. 1758) sur une rivierc à salmonidés: Le Scorff. Cybium, 9 (1): 75-87.

MANN. R.H.K. 1976. Observations on the age. growth, rcproduction and food of the pikc Esox lucius (L.) in two rivers in southern England. J. Fish Biol., 8: 179-197.

MARTINEZ, J.B. 1985. El lucio, treinta años después. Vida Silvestre, 3: 80-92.

MUNRO, W.R. 1957. The pike of Loch Choin. Freshwater and Salmon Fish. Res., 16: 16 pp.

NEUMANN, R.M., D.W. WILLIS \& S.M. SAMMONS. 1994. Seasonal growth of northern pikc (Esox lucius) in a South Dakota glacial lake. J. Freshw. Ecol.. 9 (3): 191-196.

PARAGAMIAN, V.L. 1976. Population characteristics of northern pike in the Plover river, Wisconsin. The Progressive Fish-Culturist. 38 (3): 160-163.

PENA, J.C. 1986. Introducción y expansión del lucio (Esox lucius L.. 1758) en la Península Ibérica: síntesis general y estudio de las poblaciones en la cuenca del Esla. Limnética, 2: $241-251$.

PENA. J.C., F.J. PURROY \& J. DOMINGUEZ. 1987. Primeros datos de la alimentación del lucio, Esox luciuss L. 1758, en la cuenca del Esla (España). Actas IV Congreso Español de Limnología: 271-280.

PENCZAK. T., J. LOBON-CERVIA. K. O'HARA \& H. JAKUHOWSKI. 1986. Production and food consumption by fish populations in the Pilawa and Dobrzyca rivers. North Poland. Pol. Arch. Hydrobiol., 33 (3.4): 345-372.

PIVNIKA, K. 1991. Long-term study of the growth of four fish species in the Klícava reservoir with respect to thc changes of abundance and teinperaturc. Environmentalica, 5 (1-2): 91-106.

PRIEGEL, G.R. \& D.C. KROHN. 1975. Characteristics of a northern pike spawning population. Wisconsin Dep. Nat. Res. Techn. Bull., 86:1-18.

PROKES, M. 1993. Growth of pike (Esox lucius) larvae and juveniles in the Musov reservoir. Folia Zoologica, 42 (1): 77-93.

RAAT, A. 1988. Synopsis of biological data on the northern pike Esox lucius Linnaeus, 1758. F.A.O. Fisheries Synopsis $\mathrm{N}^{\circ} 30$ Rev. 2. Roma. 178 pp.

REGUERAS, J.I. 1995. Capturas de pescadores profesionales en el río Esla. Brigecio (4-5): 235-244.

RINCON, P.A., J.C. VELASCO, N. GONZALEZ-SANCHEZ \& C. POLLO.1990. Fish assemblages in small streams in wcstern Spain: The influence of an introduced predator. Arch. Hydrobiol. 118 (1): 81-91.

SCHINDLER. O. 1975. Unsere Sübwasserfische. Kosmos Franckh. Stuttgart. 236 pp.

SNOW, H.E. \& T.D. BEARD. 1972. A ten-year study of native northern pike in Bucks lakc, Wisconsin. Wisconsin Dep. Nat. Res. Techn. Bull., No 56:1-20.

SPARRE, P. 1987. Computer programs for fish stock assessment. Length-based Fish Stock Assessment. F.A.O. Fishcries Techn. Paper 101. Roma. 218 pp.

STEEL, R.G.D. \& J.H. TORRIE. 1985. Bioestadistica: Principios fundamentales. McGraw-Hill Ed. México. 622 PP.

TABACHNICK, B.G. \& L.S. FIDELL, 1986. Using multivariate statistics. Harper and Row, Publ. New York. 746 pp.

TREASURER, J. W.. R. OWEN \& E. HOWERS. 1992. The population dynamics of pike, Esox lucius, and perch, Perca fluviatilis, in a simple predator-prey systcm. Environmental Biology of Fishes, 34: 65-78.

WEATHERLEY, A.H. 1972. Growth and ecology of fish populations. Academic Press. London. 293 pp.

WILLIS, D.W. 1989. Proposed standard length-weight equation for northern pike. North Am. J. Fish. Manag., 9: 203-208.

WOLFERT, D.R. \& T.J. MILLER. 1978. Age, growth, and food of northern pike in eastern lake Oritario. Trans. Am. Fish. Soc., 107 (5): 696-702.

WRIGHT. R.M. 1990. The population biology of pikc, Esox lucius L., in two gravel pit lakes, with special reference to early life history. .1. Fish Biol., 36: 215-229. 